\title{
Prostatic metastases and polycythemia vera on bone magnetic resonance imaging: A case report
}

\author{
YUYING LI ${ }^{1}$, WEI AN ${ }^{2}$, WEI WEI ${ }^{2}$, MIN LIU ${ }^{1}$, GUANJUN WANG ${ }^{1}$ and XU WANG ${ }^{1}$ \\ ${ }^{1}$ Cancer Center; ${ }^{2}$ Department of Urology, The First Hospital, Jilin University, \\ Changchun, Jilin 130021, P.R. China
}

Received March 18, 2014; Accepted November 21, 2014

DOI: 10.3892/ol.2014.2809

\begin{abstract}
Localized prostate cancer and polycythemia vera (PV) are rare, and can be difficult to distinguish from bone marrow metastatic prostate cancer. The present study describes a case of prostate cancer occurring with PV. Magnetic resonance imaging revealed diffusely inhomogeneous bone marrow of the pelvis and a localized prostatic mass. The bone marrow biopsy indicated erythrocytosis and leukocytosis. The patient was treated with aspirin and twice-weekly phlebotomy procedures for two weeks, followed by a radical prostatectomy. Following the surgery, the patient was continually treated with interferon- $\alpha 2 b$ and aspirin, and demonstrated no abnormalities within the one-year follow-up period. The findings of the present study may aid in the future diagnosis and treatment of patients with prostate cancer and PV.
\end{abstract}

\section{Introduction}

Prostate cancer is the most common form of cancer and the second leading cause of cancer-related mortality among males in the United States (1). In 2010, there was an incidence of $\sim 217,000$ new prostate cancer cases and 32,000 mortalities due to prostate cancer in the United States (1). Advanced-stage prostate cancer is characterized by tropism to bone, with skeletal involvement present in $\sim 90 \%$ of patients with metastatic disease $(2,3)$. Metastatic disease to bone is commonly associated with skeletal-related events, which are associated with decreased quality of life, increased pain and worsened survival (4). A bone scan is the standard method for detecting bone metastases from prostate cancer. In certain cases, additional tests, including computed tomography scan, magnetic resonance imaging (MRI) scan or bone biopsy, may be needed

Correspondence to: Miss. Xu Wang or Dr Guanjun Wang, Cancer Center, The First Hospital, Jilin University, 71 Xinmin Street, Changchun, Jilin 130021, P.R. China

E-mail: 365658791@163.com

E-mail: y13129@cumc.columbia.edu

Key words: prostate cancer, polycythemia vera, bone metastases to diagnose bone metastases. Local external beam radiotherapy, systemic radioisotope therapy, endocrine therapy, chemotherapy, and bisphosphonates and denosumab are the mainstays of treatment, as well as painkillers and other usual classical interventions (5).

Polycythemia vera (PV), a type of myeloproliferative neoplasm, is a clonal disorder characterized by unwarranted production of red blood cells, and associated with JAK2 mutations (V617F or exon 12) in almost all cases (6). It is more common in the elderly and may be symptomatic or asymptomatic. Typical signs and symptoms include itching (pruritus) and severe burning pain in the hands or feet that is frequently accompanied by a red/blue coloration of the skin. Other disease features include leukocytosis, splenomegaly, thrombohemorrhagic complications, vasomotor disturbances and a small risk of disease progression into acute myeloid leukemia (AML) or myelofibrosis (MF) (7). Diagnosis of PV is currently according to World Health Organization criteria and based on a composite assessment of clinical and laboratory features, including two major criteria (increased hemoglobin levels and presence of JAK 2 mutation) and three minor criteria (trilineage myeloproliferation in bone marrow biopsy, increased serum erythropoietin levels and endogenous erythroid colony formation in vitro) (8). The aims of therapy are to prevent the occurrence and recurrence of thrombosis; to delay (if feasible) evolution into MF or AML; and to control disease-associated symptoms (9). The treatment strategy in PV patients comprises a combination of modification of cardiovascular risk factors, antiplatelet therapy, phlebotomy and cytoreduction (9). Both of the diseases can involve bone or bone marrow, but each disease has unique changes in bone structure. As treatment options are fundamentally different between metastatic prostate cancer and PV and it is therefore important to distinguish the differences using different techniques, such as CT, MRI and bone scans. We report a case of prostate cancer and PV and our approach to differential diagnosis for these diseases using radiological imaging techniques. The patient provided written informed consent.

\section{Case report}

A 60-year-old male was admitted to The First Hospital, Jilin University (Changchun, China) in October 2012 complaining of redness on the hands and face that had been apparent for 
three months. The patient exhibited no feelings of discomfort, and reported that the skin problems appeared to be insidious. A review of the medical history revealed that the patient had experienced a lacunar infarct eight years previously, and had suffered with hypertension for $>30$ years, for which antihypertensive drugs had been taken for $\sim 20$ years. The patient did not smoke or regularly consume alcohol. Immediate family members exhibited no history of any neurological or hematological diseases.

A physical examination revealed that the patient was afebrile, with an Eastern Cooperative Oncology Group score of 0 (10), a normal heart rate and a blood pressure of 145/90 mmHg. The face, neck and hands appeared red, but no pain was reported. There was no evidence of edema or blistering of the skin. Lymphadenopathy, hepatosplenomegaly or an abnormal physical mass in the abdomen were not apparent. Digital rectal examination did not reveal any masses or enlargement of the prostate.

Hematological analysis revealed slightly elevated readings for hemoglobin $(\mathrm{Hb} ; 20.4 \mathrm{~g} / \mathrm{dl}$; normal range, $12.0-16.0 \mathrm{~g} / \mathrm{dl})$ and the hematocrit (Hct; 0.610 1/1; normal range, 0.400-0.500 1/1), and also evidence of leukocytosis [white blood cell

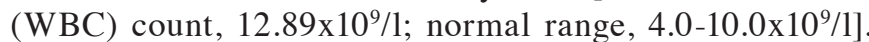
The WBC count consisted of $80 \%$ neutrophils (normal range, $50-70 \%)$, but normal levels of platelets $\left(253 \times 10^{9} / 1\right.$; normal range, $\left.100-300 \times 10^{9} / 1\right)$. The concentration of alkaline phosphatase (ALP) was slightly high (115.0 U/l; normal range, 15.0-112.0 U/l), but liver function tests appeared normal. The serum total prostate-specific antigen (TPSA) level was $10.94 \mathrm{ng} / \mathrm{ml}$ (normal range, $0-4 \mathrm{ng} / \mathrm{ml}$ ), and the serum free PSA (FPSA) level was $0.98 \mathrm{ng} / \mathrm{ml}$. As a result, the percentage of FPSA in TPSA was 9\%, which was less than the normal value of $19 \%$.

MRI of the prostate (Fig. 1) revealed a small nodule measuring $5 \times 5 \mathrm{~mm}$, which exhibited abnormal intensity signals in the right prostatic peripheral zone. The capsule of the prostate gland was continuous and complete, and no evidently enlarged surrounding lymph nodes were observed. The signal intensity of the pelvic bone marrow was diffusely inhomogeneous, but the structure of the cortical region was intact. A bone scan indicated slightly elevated radioactivity within the left ankle and right knee, but without any other abnormalities. A computed tomography scan of the lung appeared normal. A transrectal ultrasound-guided biopsy of the prostate initially revealed a well-differentiated adenocarcinoma measuring $<0.5 \times 0.1 \mathrm{~cm}$, with a Gleason score of $3+3$ (11). The bone marrow aspiration and biopsy revealed erythrocytosis and leukocytosis consisting of $12 \%$ eosinophils (normal range, $0-8 \%)$. The genetic chromosome analysis indicated the presence of a JAK2-V617F mutation, but no other abnormalities.

A diagnosis of stage IIA prostate adenocarcinoma (TNM stage, cTlaN0M0) and PV was established (12). The patient was subjected to phlebotomy procedures to remove $400 \mathrm{ml}$ of blood twice weekly for two weeks, and was treated with $100 \mathrm{mg}$ aspirin daily. Prior to surgery, the blood $\mathrm{Hb}$ level dropped to $14.1 \mathrm{~g} / \mathrm{dl}$, and the Hct to 0.47 1/1. In November 2012, a radical prostatectomy was performed by laparoscopy. Pathological examination of the surgical specimen revealed an adenocarcinoma with negative margins, but with no evidence of seminal vesicle invasion or extra-capsular extension. The

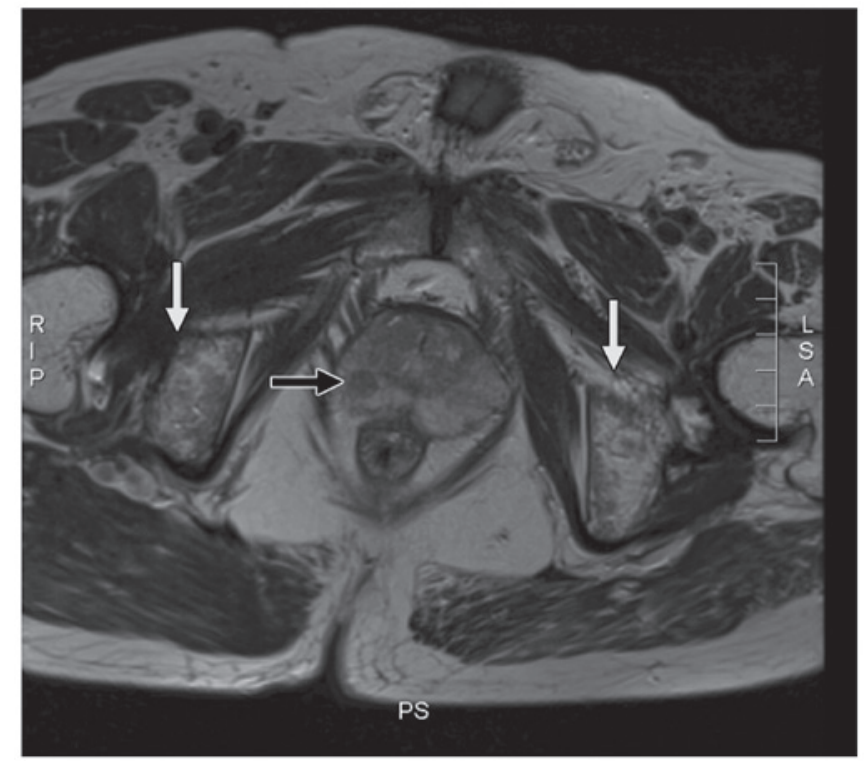

Figure 1. Magnetic resonance imaging of the prostate revealing a small nodule with abnormal signals located in the right prostatic peripheral zone (black arrow). The prostate capsule was continuous and complete, without lymph node enlargement. The signal intensity of the pelvic bone marrow was diffusely inhomogeneous (white arrows), and the structure of the cortical bone was intact.

tumor volume occupied $\sim 3 \%$ of the total prostate volume. The patient was discharged seven days after the surgery. Following surgery, the $\mathrm{Hb}$ level and Hct values gradually increased. The patient was therefore continually treated with $300 \times 10^{4}$ units of interferon- $\alpha 2 b$ (Beijing Kawin Bio-Tech Co., Ltd., Beijing, China) twice-weekly, and $100 \mathrm{mg}$ aspirin daily.

The patient was continually followed up, and the serum TPSA levels were monitored every three months. Abnormal levels of serum TPSA were not reported during the one-year follow-up period. At six months post-surgery, MRI analysis of the prostate indicated no evident abnormalities. However, there was no marked change in the atypical signal intensity of the pelvic bone marrow that was detected during the initial MRI scan. At present, the blood $\mathrm{Hb}$, Hct, platelet and WBC counts remain within the normal ranges.

\section{Discussion}

Prostate cancer is the most frequently diagnosed non-cutaneous type of cancer, and the second most common cause of cancer-related mortality affecting males in the United States. In 2010, there were an estimated 217,730 cases of newly-diagnosed prostate cancer, and at present, the disease has an estimated lifetime disease incidence in males of $20 \%$ (1). The majority of patients with advanced prostate cancer also present with bone metastases (BMs) (13-15). Patients with metastatic prostatic cancer are treated with different therapeutic strategies to those without. Therefore, it is critical to rule out the presence of metastases, such as BMs, prior to the initiation of treatment. Patients with localized prostate cancer, which is most often curable, usually benefit from radical treatment. By contrast, patients with prostate cancer and BMs are not recommended for surgery, and are usually treated with systemic chemotherapies. Several risk factors, including high 
levels of serum PSA (16-20), the presence of locally advanced tumors $(16,20,21)$, high levels of serum ALP $(16,22,23)$ and high Gleason scores (20), are believed to be associated with BMs.

In the present study, the results of the MRI scan made the diagnosis challenging. The diffusely inhomogeneous signals in the bone marrow of the pelvis, together with a small mass in the prostatic tissue, should indicate the presence of BMs. The patient exhibited histological evidence of adenocarcinoma with a Gleason score of 6, a serum PSA level of $10.94 \mathrm{ng} / \mathrm{ml}$, a slightly high level of serum ALP and a small mass in the prostatic tissue, with a continuous and complete capsule and no evidence of lymph node enlargement. Therefore, a diagnosis of localized, early-stage prostate cancer, rather than a local BM, was proposed. Previous studies have identified that prostate cancer-related BMs usually invade the cortical bone, and rarely affect the bone marrow $(24,25)$. Furthermore, BMs are characteristically accompanied by cytopenia, which ruled out the likelihood of BMs in the present case. Due to the importance of identifying the presence of BMs for correct diagnosis, a bone biopsy was performed in the present study, which identified no prostate cancer-related BMs. In addition, the bone scan did not reveal any evidence of BMs. Previous studies have demonstrated the presence of diffusely inhomogeneous signals in the bone marrow of patients with PV $(26,27)$. In the present study, the results of the laboratory tests revealed an abnormal WBC count, percentage of neutrophils, and blood $\mathrm{Hb}$ and Hct, and the presence of a JAK-2V617F mutation. Therefore, the patient was primarily diagnosed with PV and treated with aspirin, which significantly reduced the levels of blood $\mathrm{Hb}$ and $\mathrm{Hct}$ prior to the radical prostatectomy.

According to the 2013 NCCN Guidelines for Prostate Cancer, the tumor recurrence risk is intermediate, and there are no adverse features (5). Therefore, the patient was not recommended for further aggressive post-surgical therapies. Despite no significant changes identified in the signal intensity of the pelvic bone marrow during the one-year follow-up period, abnormal levels of serum PSA were not detected, which indicated no recurrence of the prostate cancer. Upon MRI analysis, prostate cancer-related BMs are often difficult to distinguish from prostate cancer cases occurring with PV. Therefore, it is important for physicians to consider these rare diseases in clinical practice. The use of bone biopsies and systemic bone scans should allow for discrimination between the two. In addition, careful analysis of MRI, with close attention to the bone structure, together with a hematological examination, should aid in the diagnosis of PV.Empiric therapies, which in the present study included the phlebotomy procedure and aspirin treatment, are valuable as they have the potential to improve clinical symptoms, restore the hematological status and improve the condition of the body for subsequent surgical treatments. The surgical removal of prostate tumors should improve the clinical symptoms of patients with prostate cancer-related PV, however, in the present study, increased values of $\mathrm{Hb}$ and Hct were observed post-surgery, which indicated an incomplete response to phlebotomy and aspirin treatment. The patient was subsequently treated with interferon- $\alpha 2 b$, which effectively controlled the symptoms of PV. Therefore, if available, interferon- $\alpha 2 \mathrm{~b}$ should be administered to patients with PV. However, the precise mechanism underlying the therapeutic action of interferon- $\alpha 2 b$ requires further investigation.

The present study described a case of prostate cancer occurring with PV. The simultaneous presence of these diseases should be recognized, and careful analysis of diagnostic imaging, such as MRI, should be performed. Furthermore, risk factors for BMs should be considered following the detection of abnormal intensity signals in the pelvis of patients with prostate cancer. To the best of our knowledge, this was the first case study to describe the systemic medical history and treatment of a patient with prostate cancer occurring with PV. The findings of the present study may aid in the future diagnosis and treatment of patients with prostate cancer and PV.

\section{References}

1. Jemal A, Siegel R, Xu J and Ward E: Cancer statistics, 2010. CA Cancer J Clin 60: 277-300, 2010.

2. Scher HI, Morris MJ, Kelly WK, Schwartz LH and Heller G: Prostate cancer clinical trial end points: 'RECIST'ing a step backwards. Clin Cancer Res 11: 5223-5232, 2005.

3. Jacobs SC: Spread of prostatic cancer to bone. Urology 21: 337-344, 1983.

4. DePuy V, Anstrom KJ, Castel LD, Schulman KA, Weinfurt KP and Saad F: Effects of skeletal morbidities on longitudinal patient-reported outcomes and survival in patients with metastatic prostate cancer. Support Care Cancer 15: 869-876, 2007.

5. National comprehensive cancer network: NCCN Clinical Practice Guidelines in Oncology: Prostate cancer. http://www. nccn.org/professionals/physician_gls/pdf/prostate.pdf. Accessed May 23, 2013.

6. Tefferi A: Polycythemia vera and essential thrombocythemia: 2013 update on diagnosis, risk-stratification, and management. Am J Hematol 88: 507-516, 2013.

7. Passamonti F: How I treat polycythemia vera. Blood 120: 275-284, 2012.

8. Spivak JL and Silver RT: The revised World Health Organization diagnostic criteria for polycythemia vera, essential thrombocytosis, and primary myelofibrosis: an alternative proposal. Blood 112: 231-239, 2008.

9. Barbui T, Barosi G, Birgegard G, et al: Philadelphia-negative classical myeloproliferative neoplasms: critical concepts and management recommendations from European LeukemiaNet. J Clin Oncol 29: 761-770, 2011.

10. Oken MM, Creech RH, Tormey DC, et al: Toxicity and response criteria of the Eastern Cooperative Oncology Group. Am J Clin Oncol 5: 649-655, 1982.

11. Gleason DF and Mellinger GT: Prediction of prognosis for prostatic adenocarcinoma by combined histological grading and clinical staging. J Urol 111: 58-64, 1974.

12. Edge SB, Byrd DR, Compton CC, Fritz AG, Greene FL and Trotti A (eds): Prostate. In: AJCC Cancer Staging Manual. 7th edition. Springer, New York, pp457-468, 2010.

13. Tombal B and Lecouvet F: Modern detection of prostate cancer's bone metastasis: Is the bone scan era over? Adv Urol 2012: 893193, 2012

14. Nørgaard M, Jensen A $\varnothing$, Jacobsen JB, Cetin K, Fryzek JP and Sørensen HT: Skeletal related events, bone metastasis and survival of prostate cancer: a population based cohort study in Denmark (1999 to 2007). J Urol 184: 162-167, 2010.

15. Carlin BI and Andriole GL: The natural history, skeletal complications, and management of bone metastases in patients with prostate carcinoma. Cancer 88 (Suppl 12): 2989-2994, 2000.

16. Briganti A, Passoni N, Ferrari M, et al: When to perform bone scan in patients with newly diagnosed prostate cancer: external validation of the currently available guidelines and proposal of a novel risk stratification tool. Eur Urol 57: 551-558, 2010.

17. Lai MH, Luk WH and Chan JC: Predicting bone scan findings using sPSA in patients newly diagnosed of prostate cancer: feasibility in Asian population. Urol Oncol 29: 275-279, 2011.

18. Ho CC, Seong PK, Zainuddin ZM, Abdul Manaf MR, Parameswaran M and Razack AH: Retrospective study of predictors of bone metastasis in prostate cancer cases. Asian Pac J Cancer Prev 14: 3289-3292, 2013. 
19. Thompson I, Thrasher JB, Aus G, et al; AUA Prostate Cancer Clinical Guideline Update Panel: Guideline for the management of clinically localized prostate cancer: 2007 update. J Urol 177: 2106-2131, 2007.

20. Heidenreich A, Aus G, Bolla M, et al; European Association of Urology: EAU guidelines on prostate cancer. Eur Urol 53: 68-80, 2008.

21. Koutsopoulos AV, Dambaki KI, Datseris G, Giannikaki E, Froudarakis M and Stathopoulos E: A novel combination of multiple primary carcinomas: urinary bladder transitional cell carcinoma, prostate adenocarcinoma and small cell lung carcinoma - report of a case and review of the literature. World $\mathbf{J}$ Surg Oncol 3: 51, 2005.

22. Têtu B, Ro JY, Ayala AG, Johnson DE, Logothetis CJ and Ordonez NG: Small cell carcinoma of the prostate. Part I. A clinicopathologic study of 20 cases. Cancer 59: 1803-1809, 1987.
23. Rubenstein JH, Katin MJ, Mangano MM, Dauphin J, Salenius SA, Dosoretz DE and Blitzer PH: Small cell anaplastic carcinoma of the prostate: seven new cases, review of the literature, and discussion of a therapeutic strategy. Am J Clin Oncol 20: 376-380, 1997

24. Seo Y, Shuke N, Yamamoto W, Usui K and Aburano T: Sub-super bone scan caused by bone marrow involvement of prostate cancer. Ann Nucl Med 13: 351-354, 1999.

25. Tachev S, Ormanov I and Slavov CH: Cancer of the prostate with metastasis to the bone marrow. Khirurgiia (Sofiia) 39: 96-97, 1986 (In Bulgarian).

26. Steiner RM, Mitchell DG, Rao VM, et al: Magnetic resonance imaging of bone marrow: diagnostic value in diffuse hematologic disorders. Magn Reson Q 6: 17-34, 1990.

27. Birchall JD and O'Connor RA: Prostatic metastases versus polycythemia on bone imaging. Clin Nucl Med 29: 375-377, 2004. 